\title{
Induction of Male-Sterility by Controlling of Gibberellin Biosynthesis in Rice (Oryza sativa)
}

\author{
Hyun Kyung Bae ${ }^{1,2}$, Sung Aeong $\mathrm{Oh}^{1}$, Soon Ki Park ${ }^{1}$ * \\ ${ }^{1}$ School of Applied Biosciences, Kyungpook National University, Daegu 41566, Korea \\ ${ }^{2}$ Department of Southern Area Crop Science, NICS, RDA, Miryang 50424, Korea
}

\begin{abstract}
Gibberellins (GAs) are important hormones at every plant developmental stage including vegetative and reproductive. It is reported in many plant species that many dwarfism and male sterile mutants have defective GA biosynthesis genes. RNA interference (RNAi) technology can reduce expression of specific genes at the transcription level. Also, promoters can control the spatial and temporal expression of genes. Specific promoters containing the RNAi vector can control specific genes' spatial and temporal expression at the transcriptional level. In this study, at first, five anther specific promoters were chosen and verified by examining their tissue specific expression using promoter::GUS transgenic analysis. RNAi vectors, which contain five anther specific promoters and one constitutive promoter fused to GA biosynthesis related genes, were constructed for controlling of GA biosynthesis in stem and floral tissue. OsAct1 promoter containing OsGA20ox1 and OsGA3ox2 RNAi T1 plants showed reduced gene expression and short plant height phenotype compare to wild type plant. OsGA20ox and OsGA3ox RNAi transformants also showed reduced fertility or sterile phenotype.
\end{abstract}

Keywords Dwarfism, Gibberellic acid, RNA interference, Pollen specific promoter, Male sterility

\section{INTRODUCTION}

The developmental stage of male reproductive organ in flowering plants begins with the establishment of the stamen (Ma 2005). The stamen consists of two distinct structures, the anther and filament. The anther is composed of highly specialized tissues for pollen generation while the filament provides support for the anther. Two sequential steps compose the male gametophytic development, microsporogenesis and microgametogenesis. Itoh et al. (2005) classified anther development into 8 stages. Initially, the anther conformation changes from ovoidal into fourcornered shape and the archesporial cells (ACs) initiate at the hypodermal layer of the anther. The ACs differentiates into primary sporogenous cells (PSCs) and primary parietal cells (PPCs). After several rounds of division, the anther wall layer is formed. PPCs will continue to divide periclinally to generate the endothecium, a middle layer and a tapetum layer and will expand anticlinally. After the anther wall is completed, the PMCs undergo meiosis to produce haploid gametes. The microspore increases in size and undergoes an asymmetric cell division (pollen mitosis I) which produces two cells, the vegetative cell and generative cell. The generative cell undergoes symmetric cell division to produce two sperm cells before pollen tube germination. Finally, the tricellular pollen produced during male gametogenesis contains two sperm cells and one vegetative cell that are required for double fertilization of the egg cell and polar nuclei.

The flower structure of rice is relatively different from

Received October 30, 2017; Revised December 4, 2017; Accepted December 5, 2017; Published March 1, 2018

*Corresponding author Soon Ki Park, psk@knu.ac.kr, Tel: +82-53-950-7751, Fax: +82-53-958-6880 
other cereal crops, like wheat and maize. The rice inflorescence is characterized by the presence of one spikelet that contains one floret enclosed by a pair of empty glumes (Zhang and Wilson 2009). Additionally, the rice floret is bisexual and the floral organs are asymmetrically arranged comprising of one lemma and one palea (first outer whorl), two lodicules (second whorl), six stamens (third whorl) and one pistil (fourth innermost whorl) (Yamaguchi et al. 2006; Hirano et al. 2008; Zhang and Wilson 2009).

Microarray analyses of the four stages (pre-meiotic, meiotic, single celled anther and trinucleate pollen) of anther development identified 1,000 genes specifically expressed in anther (Deveshwar et al. 2011). In a similar study by Fujita et al. (2010), the stages of rice reproductive development were dissected and gene expression levels were determined using microarray technology. The 2,593 probe sets were identified on developing anther, whereas only a few were detected in other reproductive stages like fertilization and embryonic stages. Therefore, proper understanding and knowledge of reproductive development processes are very important in the field of plant breeding, especially for hybrid seed production.

Generally, a gene's spatial and temporal expression pattern is determined by its own promoter. Use of an appropriate tissue specific promoter is a critical factor to control gene expression at specific stage and organ-specific manners. In rice, many kinds of pollen/anther/tapetumspecific genes with their promoters have been reported using traditional approaches and recently have been identified via transcriptome study through microarray analysis (Endo et al. 2004; Suwabe et al. 2008; Tang et al. 2010). Most of the genes that are expressed at early stage of microspore development are usually tapetum-specific since their cells usually play roles in leading the initial development of microspore. However, binucleate pollen stage at the beginning of tapetum cell degradation stage, gene expression patterns are rapidly changed. Expressions of tapetum specific genes disappeared and pollen specific genes begin to emerge at that point. The tapetum specific gene $O s c 4$ is expressed at the early uninucleate microspore stage for a short time (Tsuchiya et al. 1992; Tsuchiya et al. 1994). Osg $6 b$ show strong expression at uninucleate microspore stage and it is gradually reduced to trinucleate pollen stage
(Tsuchiya et al. 1992; Tsuchiya et al. 1994). In cases of pollen-specific genes, $O S S C P 1$ and $O S S C P 2$ belonging to a multi-gene family, $O S S C P$, are expressed at the late stage of pollen development from tricelluar pollen stage to pollen tube germination (Park et al. 2006). OsIPA was identified as an indica rice derived pollen specific gene, is expressed at the late pollen development stage like $O S S C P$ family (Gupta et al. 2007).

Gibberellins (GAs) diterpenoids that are based on a tetracyclic ent-gibberellane skeleton act as a growth regulator during plant development. Generally, GAs have an active role in plant vegetative development and also control the development of reproductive organs such as anther, tapetum and pollen. Generally, low GA contents can cause the delay or elimination of anthesis. Some studies showed that GA deficient or signaling mutant can induce male-sterile rice (Sakamoto et al. 2001; Kaneko et al. 2003). Also in Arabidopsis, GA-deficient mutant showed male-sterile phenotype with normal female organs (Koornneef and van der Veen 1980). GAMYB is widely known as GA signaling factor and a knout out mutant of rice $O S G A M Y B$ showed abnormal development of floral organs, shrunken and whitened anther, and delayed heading time in rice (Gubler et al. 1995; Kaneko et al. 2003). Additionally, GA- insensitive and defective mutants showed low fertilization by pollen viability and pollen tube growth (Chhun et al. 2007). Most GA-related studies in rice have been focused on plant vegetative aspects such as stem growth. Many research groups also reported that GA-related mutants can be cause of severe male-sterility in rice (Sakamoto et al. 2001; Oikawa et al. 2004; Sakamoto et al. 2004; Qiao et al. 2007). Interestingly, the most famous GA biosynthesis- related gene, knock-out mutant $s d 1$ and $d 18$, did not show any fertility changes (Sakamoto et al. 2004). Therefore, $s d l$ has been used as a critical gene for semi-dwarf rice breeding. This result, nevertheless, also provides another opportunity to study rice flower development with GA biosynthesis related genes - OsGA20ox gene including sd1, GA20ox2 with four iso-genes, OsGA3ox gene including $d 18$ and GA3ox2 with 2 iso-genes. It means that except $s d 1$ and $d 18$, other genes have possibility of controlling flower development. 
In this study we constructed six vectors containing five anther/pollen specific promoters and one constitutive promoter fused with GUS::GFP to verify the tissue specific expression of promoters. In addition, twenty kinds of RNAi vector were constructed to induce a male-sterile rice, which harbored five anther/pollen specific promoters of Osc4, Osg6b (Tsuchiya et al. 1994), OsSCP1, OsSCP2 (Park et al. 2006) and OsIPA (Gupta et al. 2007), one constitutive promoter of OsAct1, and four GA biosynthesis related genes such as OsGA20ox1, OsGA20ox3, OsGA3ox1 and OsGA3ox2; were constructed to induce a male-sterile rice. As a result, each GA-RNAi $\mathrm{T}_{0}$ lines showed lower fertility than control $\mathrm{T}_{0}$ lines but had similar grain numbers. Several GA-RNAi progenies showed short culm length trait as expected.

\section{MATERIALS AND METHODS}

\section{Cloning of rice promoters}

The promoter sequence information of six rice genes, Osc4 (1,638-bp promoter), Osg6b (1,671-bp promoter), OSSCP1 (2,033-bp promoter), OSSCP2 (952-bp promoter), OsIPA (1,887-bp promoter) and OsAct1 (1435-bp promoter) were obtained through reference papers (McElroy et al. 1990; Tsuchiya et al. 1994; Park et al. 2006; Gupta et al. 2007). The japonica rice cv. Dongjin genomic DNA was used as template for PCR reaction (Table 1). The condition for PCR reaction was 30 cycles of $94^{\circ} \mathrm{C} 30$ seconds, $55^{\circ} \mathrm{C}$
30 seconds, and $72^{\circ} \mathrm{C} 2$ minutes, using Pfu-X DNA polymerase (Solgent, Korea). PCR product of Osc4 promoter (hereafter pro) and Osg $6 b$ pro were cloned into pGEMT-easy vector (Promoga, USA) and PCR product of OsSCP1 pro, OsSCP2 pro, OsIPA pro and OsAct1 pro were cloned into T-Blunt vector (Solgent, Korea).

\section{Construction of promoter-GUS::GFP fused vector}

For the LR cloning, promoters cloned into pGEMT-easy and T-Blunt vector were used as a template DNA for PCR amplification. The $1^{\text {st }} \mathrm{PCR}$ reaction condition was 10 cycles of $94^{\circ} \mathrm{C} 30$ seconds, $55^{\circ} \mathrm{C} 30$ seconds, and $72^{\circ} \mathrm{C} 3$ minutes using Pfu-X DNA polymerase (Solgent, Korea). The $2^{\text {nd }} \mathrm{PCR}$ was performed using an Attb primer pair and the PCR reaction condition was 5 cycles of $94^{\circ} \mathrm{C} 30$ seconds, $45^{\circ} \mathrm{C} 30$ seconds, and $72^{\circ} \mathrm{C} 1$ minute and 20 cycles of $94^{\circ} \mathrm{C} 30$ seconds, $55^{\circ} \mathrm{C} 30$ seconds, and $72^{\circ} \mathrm{C} 3$ minutes. PCR products were cloned into a pDONR201 entry vector using BP clonase II mix. The promoter inserted entry vectors were combined with pKGWFS7 destination vector for promoter analysis using LR clonase II mix.

\section{Cloning of RNAi trigger region}

The japonica rice cv. Dongjin DNA was used for PCR reaction. RNAi trigger regions from four rice genes OsGA3ox1, OsGA3ox2, OsGA20ox1 and OsGA20ox3 were PCR amplified using gene specific primers (Table 2). The PCR reaction conditions for OsGA3oxl and OsGA3ox 2 were 30 cycles of $94^{\circ} \mathrm{C} 30$ seconds, $55^{\circ} \mathrm{C} 30$

Table 1. Primer sequences for rice promoter cloning.

\begin{tabular}{|c|c|c|}
\hline Promoter & Primer sequences $\left(5^{\prime} \rightarrow 3^{\prime}\right)$ & Size $(b p)$ \\
\hline \multirow[t]{2}{*}{ Osc4 pro } & F: AAGCTTCAGACCGCTGGTCAGTGGA & 1,638 \\
\hline & R: CTCGAGCTCCTGTGCTTCTTCAGAC & \\
\hline \multirow[t]{2}{*}{ Osg6b pro } & F: AAGCTTTTGGTTAAAACCCTCAGGT & 1,671 \\
\hline & R: CTCGAGCTTGAGCTAGCTTAATTAG & \\
\hline \multirow{2}{*}{ OsSCP1 pro } & F: AAAACTCGAGTCTGTTGTTCTACCCAATATTGTTAC & 2,033 \\
\hline & R: TTTTATTAATTAGCACCGTTTCTATTGAGGGTAAC & \\
\hline \multirow[t]{2}{*}{ OsSCP2 pro } & F: TAGCACCGTTTATGCTGAGCAGAATTGGTG & 952 \\
\hline & R: ACTGGGTCGACTTGGGCCGGGGAGGAGAGA & \\
\hline \multirow[t]{2}{*}{$O s I P A$ pro } & F: AAAAAAGCTTGTGTCGACCTCGGCATCCTCGGCG & 1,887 \\
\hline & R: TTTTCTCGAGGTCGCTTTTATTTGGCTTGGTGTGTTT & \\
\hline \multirow{2}{*}{ OsAct1 pro } & F: AAAAAAGCTTTAGCTAGCATACTCGAGGTCATTCATATGC & 1,435 \\
\hline & R: TTTTATTAATGATATCCTCGGCGTCAGCCATC & \\
\hline
\end{tabular}


Table 2. Primer sequences for the cloning of RNAi trigger fragments.

\begin{tabular}{clc}
\hline \hline Genes & \multicolumn{1}{c}{ Primer sequences $\left(5^{\prime} \rightarrow 3^{\prime}\right)$} & Size $(\mathrm{bp})$ \\
\hline OsGA20ox1 & F: AAAAAGCAGGCTGCTGTCGTTCCGGTACTCATCGG & 457 \\
& R: AGAAAGCTGGGTTGCGGTACCTGGCGTTGGAGAG & \\
OsGA20ox3 & F: AAAAAGCAGGCTCAGGAGTACTGCGACGTGATGACG & 422 \\
& R: AGAAAGCTGGGTGGGTTGAGGAAGAAGGTGAGCGAC & \\
OsGA3ox1 & F: GGATCCCTAGGTGTCCAGATCCA & 272 \\
& R: CTCGAGGAAGTAGGGCATGGAG & \\
Fs $:$ GGATCCGTCGCACTTGAAGAACC & R: CTCGAGGGAGGAAGGGGAGAAGG & 409 \\
\hline
\end{tabular}

seconds, and $72^{\circ} \mathrm{C} 1$ minute using Pfu-X DNA polymerase (Solgent, Korea). PCR products of OsGA3oxl and OsGA3ox2 were double digested with HindIII/XhoI and cloned into pENTR donor vector. OsGA20oxl and OsGA20ox3 were cloned into pDONR201 by using BP reaction. The $1^{\text {st }}$ PCR reaction condition of OsGA20oxl and $O s G A 20 o x 3$ was $94^{\circ} \mathrm{C} 30$ seconds, $55^{\circ} \mathrm{C} 30$ seconds, and $72^{\circ} \mathrm{C} 1$ minute and then the $2^{\text {nd }} \mathrm{PCR}$ was performed using an Attb primer pair. The $2^{\text {nd }} \mathrm{PCR}$ reaction condition was 5 cycles of $94^{\circ} \mathrm{C} 30$ seconds, $45^{\circ} \mathrm{C} 30$ seconds, and $72^{\circ} \mathrm{C} 1$ minute and 20 cycles of $94^{\circ} \mathrm{C} 30$ seconds, $55^{\circ} \mathrm{C} 30$ seconds, and $72^{\circ} \mathrm{C} 1$ minute. PCR products were cloned into pDONR201 vector using BP clonase II mix (Invirogen, USA).

\section{Promoters inserted RNAi destination vectors}

Promoters (Osc4 pro, Osg6b pro, OsSCP2 pro, OsIPA pro and $O s A c t 1$ pro) that cloned into T-Blunt vector were digested with HindIII/XhoI. The digested fragments were then cloned into promoter insertion sites in $\mathrm{pK} 7$ gwiwgL RNAi vector (http://www.psb.ugent.be/gateway), which was digested with same restriction enzymes. OSSCPI promoter was cloned into $\mathrm{pK} 7 \mathrm{gwiwgL}$ RNAi vector by double-digestion with $\mathrm{XhoI} / A s e \mathrm{I}$. The resulting $\mathrm{pK} 7 \mathrm{gwiwgL}$ RNAi destination vectors containing each promoter were combined with donor vector (pENTR; OsGA3oxl, OsGA3ox2: pDONR201; OsGA20ox1, OsGA20ox3) using LR clonase II (Invitrogen, USA).

\section{Agrobacterium mediated rice transformation}

The japonica rice cv. Dongjin was used as the material for transformation. Transgenic rice plants were generated using Agrobacterium-mediated transformation of rice calli, as described by Toki et al. (2006). The transgenic rice plants were cultivated in the GMO experimental field of Kyungpook National University (Gunwi, Gyeongsangbukdo province).

\section{DNA extraction and T-DNA confirm PCR}

Rice genomic DNA was extracted using Cetyltrimethylammonium bromide (CTAB) DNA isolation method as described by Ronald and Chen (1999). The presence of T-DNA in genome of transgenic plants was confirmed by PCR amplification using Attb primers. PCR condition was 30 cycles of $94^{\circ} \mathrm{C} 30$ seconds, $45^{\circ} \mathrm{C} 30$ seconds, and $72^{\circ} \mathrm{C}$ 1 minute.

\section{Histochemical GUS assay}

Anther from $T_{0}$ transgenic plants were collected at 1-2 weeks before heading and used for histochemical $\beta$ glucuronidase (GUS) assay. The GUS staining solution was $100 \mathrm{mM}$ sodium phosphate buffer, $\mathrm{pH} 7.0,20 \%$ methanol, $0.1 \%$ triton $\mathrm{X}-100,0.5 \mathrm{mM} \mathrm{K}_{3} \mathrm{~F}_{2}(\mathrm{CN})_{6}$ and $1 \mathrm{mM}$ $\mathrm{X}$-GlcA (5-bromo-4-chloro-3-indoxyl- $\beta$-D-glucuronic acid). Five spikelets were incubated overnight at $37^{\circ} \mathrm{C}$ and then chlorophyll was removed by washing with $70 \%$ ethanol.

\section{RNA extraction and semi-quantitative RT-PCR}

Plant total RNA was extracted using Qiazol RNA extraction buffer (Qiagen, USA). RNA was extracted from 4-week old $T_{1}$ plants from each semi-dwarf line. Two micrograms of total RNA were used to generate first-stand cDNA through RT-PCR by using Diastar-RTase kit (Solgent, Korea) with oligo(dT) 18 primer (Genotech, 
Korea). Primer sequences for RT-PCR analysis of OsGA20ox 1 and OsGA3ox2 genes were designed based on information provided by Sakamoto et al. (2004). The total volume of single reaction was $20 \mu \mathrm{L}$ containing $1 \mu \mathrm{L}$ cDNA, 1X PCR buffer, $0.2 \mathrm{mM}$ dNTPs, $0.2 \mu \mathrm{M}$ primer and 1 unit of Diastar-Taq DNA polymerase (Solgent, Korea). The reaction condition was 35 cycles of $94^{\circ} \mathrm{C} 30$ seconds, $45^{\circ} \mathrm{C} 30$ seconds, and $72^{\circ} \mathrm{C} 1$ minute. Relative quantity of RT-PCR product was calculated with the used of Image Lab software (Bio-Rad Laboratories).

\section{Fertility analysis}

To estimate spikelet fertility, five panicles were selected randomly from each $T_{0}$ plant at maturity and their grain numbers were counted. Sunken grains in the distilled water were counted as fertile; seed and floated grains were counted as sterile. The fertility rate (FR) describes the fraction of grains with successfully seed sets to the total number of grains from 5 panicles of $\mathrm{T}_{0}$ plant; Thus, FR (\%)= (fertile grain/total grain) $\times 100$.

\section{Hydroponic culture of rice seedling}

Rice seeds were soaked in seed disinfectant for a day and soaked seeds were incubated at $28^{\circ} \mathrm{C}$ in dark for 24 hours after removing the disinfectant. Germinated seeds were sowed on sterilized sand and were grown for a week under a condition of $32^{\circ} \mathrm{C}, 14$-hour light and $28^{\circ} \mathrm{C}, 10$-hour dark. Twenty well-grown, same size rice seedlings were planted on rice hydroponic solution (Yoshida et al. 1976). Rice seedlings were grown for 4 weeks at a culture condition of $32^{\circ} \mathrm{C}, 14$-hour light and $28^{\circ} \mathrm{C}, 10$-hour dark in growth chamber. Hydroponic solution was changed once a week. Within twenty seedlings, three were collected for RNA extraction and the others were used for GA contents analysis.

\section{RESULTS}

\section{Construction of promoters-GUS::GFP fused vector} for the analysis of anther/pollen specific promoters

To verify the tissue-specificity of promoters, pKGWFS7 promoter analysis vector was used. pKGWFS7 is the promoterless-vector system fused GUS/GFP reporter genes containing attR 1 and attR2 sites at the promoter region for LR cloning. Six promoters; Osc4 pro, Osg $6 b$ pro, OsSCP1 pro, OsSCP2 pro, OsIPA pro and OsActl pro, which were cloned to the pDONR201 were inserted into pKGWFS7 by LR cloning. Previously, it has been reported that, Osc4 pro and $O s g 6 b$ pro were characterized as tapetum-specific promoters, and $O S S C P 1$ pro, OsSCP2 pro and OSIPA pro were pollen-specific promoters (Tsuchiya et al. 1994; Park et al. 2006; Gupta et al. 2007). Also, OsAct1 pro is widely used constitutive promoter as a control. Consistent with previous reports, all analyzed $\mathrm{T}_{0}$ transgenic plants exhibited strong GUS activity at the anther except $O S S C P 2$ pro. As we expected, OsAct1 pro induced strong expression at whole spikelet tissue (Fig. 1).

\section{RNAi vector construction for the suppression of GA biosynthesis}

In the GA biosynthesis pathway, many different kinds of enzymes are involved. Among these enzymes, GA 20oxidase and GA 3-oxidase are known key enzymes which catalyze the inactive GAs to active GAs. There are four GA 20-oxidase genes; (OsGA20ox1, OsGA20ox2, OsGA20ox3, and $O s G A 20 o x 4$ ) and two GA 3-oxidase genes (OsGA3ox 1 and $O s G A 3 o x 2$ ) in rice. All OsGA20ox genes are expressed in panicles at different levels. OsGA20ox1, OsGA20ox2,

A

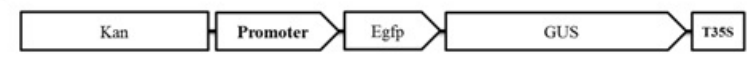

B

$1 \mathrm{~kb}$

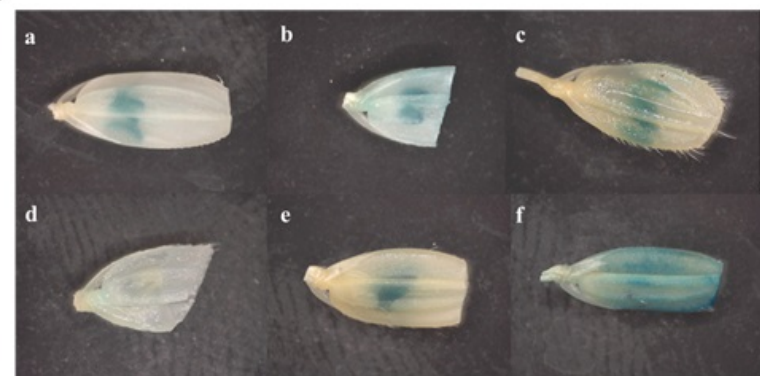

Fig. 1. Histochemical GUS analysis of rice flower. (A) Structure of pKFWFS7 destination vector carrying promoter-GUS::GFP gene fusion. (B) Results of GUS analysis. (a) Osc4 pro, (b) Osg6b pro, (c) OsSCP1 pro, (d) $O s S C P 2$ pro, (e) OsIPA pro, (f) OsAct1 pro. 
and $O s G A 20 o x 4$ are expressed in all vegetative organs at different levels but OsGA20ox3 is only expressed at panicle. Like OsGA20ox, OsGA3ox2 is expressed in all organs but $O s G A 30 x 1$ is only expressed at panicle (Itoh et al. 2001; Kaneko et al. 2003; Sakamoto et al. 2004; Hirano et al. 2008). With this result, OsGA20ox3 and OsGA3oxI are expected to be the floral tissue specific genes. The $s d l$ (OsGA20ox2) and $d 18$ (OsGA3ox2) are also widely known dwarf mutants caused by GA deficiency (Itoh et al. 2001; Spielmeyer et al. 2002). For controlling of GA biosynthesis at the anther/pollen development stage in rice, two OsGA20ox iso-genes (OsGA20oxl and OsGA20ox3) and two OsGA3ox iso-genes (OsGA3ox 1 and OsGA3ox2) were

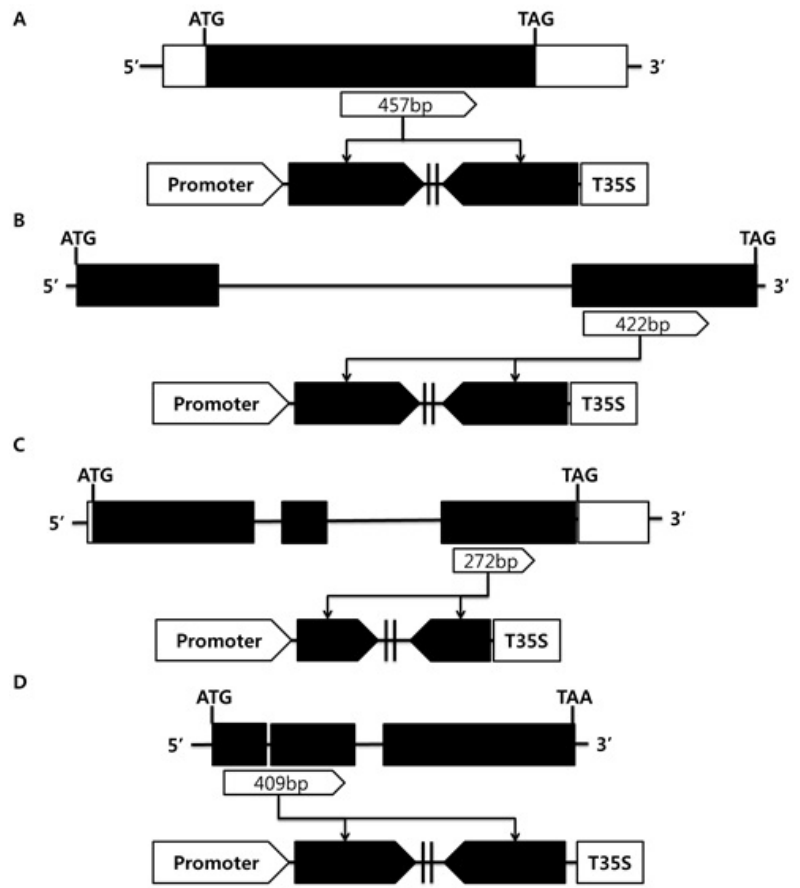

Fig. 2. Structure of $O S G A 200 x$ and $O S G A 3 o x$ genes and their RNAi trigger regions used for construction of RNAi vectors. (A) OsGA20oxl (457 bp), (B) OsGA20ox3 (422 bp), (C) OsGA3oxl (272 bp), (D) OsGA3ox2 (409 bp). chosen in order to construct the RNAi vector. Also, promotors of previously reported five anther/tapetum/pollenspecific genes (Osc4, Osg6b, OsSCP1, OsSCP2, OsIPA) and one constitutive expressed gene OsAct1 were used to control tissue specific expression. Donor vector containing PCR amplified RNAi trigger fragments OsGA20oxl (457 bp), OsGA20ox3 (422 bp), OsGA3oxl (272 bp) and OsGA3ox2 (409 bp) were combined with pK $7 \mathrm{gwiwgL}$, and the resulting twenty RNAi destination vectors were constructed to generate rice transgenic plants (Fig. 2).

\section{Generation of semi-dwarf mutant rice via transformation using RNAi vector}

GA plays an important role in plant stem growth. Especially two GA biosynthesis-related genes, OsGA20ox2 and OsGA3oxl, are known to the major gene in rice vegetative growth. Two rice mutants, $s d l$ and $d 18$, which have mutations in these, were widely known to exhibit unique mutant phenotype of dwarfism due to a defective GA-biosynthesis pathway. It has also been reported that OsGA20oxl was involved in rice stem growth through GA processes. Some studies showed the possibility of semidwarfism induced through biotechnological approach via controlling of OsGA20ox2 or OsGA3ox2 (Itoh et al. 2002; Qiao et al. 2007).

To examine whether RNAi constructs of OsGA20oxI and OsGA3ox2 affected GA biosynthesis in rice, transgenic lines were generated and analyzed on plant height and gene expression. If the RNAi vectors used in this study worked properly, we expected that resulting transgenic plants would be semi-dwarf as previously reported (Itoh et al. 2002; Oikawa et al. 2004; Qiao et al. 2007). As we expected, transgenic lines of OsAct1 pro-OsGA20ox1 and OsAct1 pro-OsGA3ox 2 RNAi in $\mathrm{T}_{1}$ generation, showed semi-dwarf phenotype but not in OsGA20ox3 RNAi transgenics. $\mathrm{T}_{1}$ transgenic progeny plants generated by RNAi vector of OsAct1 pro-

Table 3. Summary of plant heights of semi-dwarf lines.

\begin{tabular}{|c|c|c|c|c|c|c|c|c|}
\hline Line & Dongjin & $200 \times 1-1$ & $200 \times 1-2$ & $200 \times 1-3$ & $30 \times 2-1$ & $30 \times 2-2$ & $30 \times 2-3$ & $30 \times 2-4$ \\
\hline lant height $(\mathrm{cm})$ & $100.8 \pm 8.3^{\mathrm{a}, \mathrm{z})}$ & $79.2 \pm 9.9^{\mathrm{bc}}$ & $85.6 \pm 5.0^{\mathrm{b}}$ & $74.3 \pm 5.9^{\mathrm{c}}$ & $75.3 \pm 3.4^{\mathrm{bc}}$ & $82.4 \pm 4.7^{\mathrm{bc}}$ & $84.1 \pm 5.3^{\mathrm{b}}$ & $80.9 \pm 10.0^{\mathrm{bc}}$ \\
\hline
\end{tabular}

\footnotetext{
${ }^{2)}$ Values with the same letter in a column are not significantly different at $5 \%$ probability level as determined by DMRT.
} 
OsGA20ox 1 gene exhibited approximately $20 \%$ shorter plant height which ranged from 74.3 $\pm 5.9-85.6 \pm 5.0 \mathrm{~cm}$ compared to cv. Dongjin wildtype plant with average height of $100.8 \pm 8.3 \mathrm{~cm} . \mathrm{T}_{1}$ transgenics from OsAct 1 pro-OsGA3ox2 RNAi vector also showed similar pattern in height with the ranges of 75.3 $\pm 3.4-84.1 \pm 5.3 \mathrm{~cm}$ (Table 3). Other transgenics containing specific promoter had not shown decreased height phenotype but some lines showed reduced fertility (data not shown).

Hence, it suggested that expression of these genes, OsGA20oxl and OsGA3ox2, were required for rice stem growth, strongly indicated that they played a role in GA biosynthesis pathway in rice. Furthermore, these results provide the possibility that these RNAi constructs can be applied to control GA biosynthesis in specific organs such as anther or pollen for the induction of male sterility.

\section{Analysis of gene expression}

Many GA-related studies previously reported the expression of OsGA20ox 1 and $O S G A 30 \times 2$ in all tissues and all stages of rice growth period (Kaneko et al. 2003; Sakamoto et al. 2004; Hirano et al. 2008). OsGA3ox2 is an especially well-known major GA3ox gene in rice vegetative growth. Although OsGA20ox2, among GA20ox 4 isogenes, has been known as a major GA20ox in rice vegetative growth and $O S G A 20 o x 1$ has weaker expression than OsGA20ox2, OsGA20ox1 also has the significant effect on rice stem growth (Oikawa et al. 2004).

To investigate the down-regulation of target gene expression of OsAct1 pro-OsGA20oxl and OsAct1 proOsGA3ox2 RNAi transgenic plants, RT-PCR was performed using total RNA extracted from 4-week old rice stem tissues. Consistent with the results on plant heights, 4-week old seedlings also showed semi-dwarf phenotype (data not

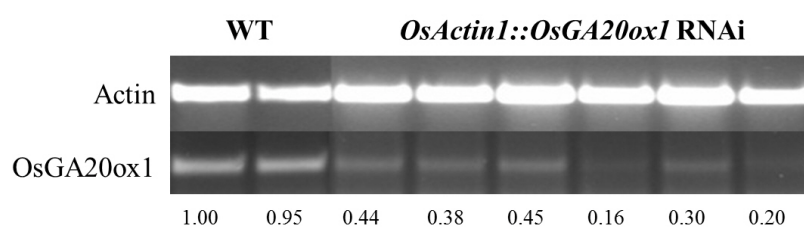

Fig. 3. RT-PCR result of OsAct1 pro-OsGA20ox1 semidwarf mutant lines. The numbers below gel photo are relative brightness value of the PCR band. shown). We confirmed that all plants contained T-DNA copies in their genome. The expression of the target gene in all OsAct1 pro-OsGA20ox1 RNAi lines tested was relatively weaker compared to wildtype (Fig. 3). However, in case of $O s G A 3 o x 2$ RNAi lines, expression levels were variable among transgenic lines (Fig. 4). Since the expression of $O s G A 3 o x 2$ in wild type plants was significantly weak, it's difficult to evaluate.

Among the transgenic lines generated from OsGA3ox2, we observed the typical semi-dwarf phenotype in two lines with similar expression levels compared to wildtype. Even though the increased expression level does not explain the observed phenotype on seedling height, these results suggested that RNAi constructs, to a certain extent, influenced the expression level of OsGA3ox2.

\section{Generation of sterile rice regulation of $O s G A 200 x$ and OsGA3ox expression.}

Our experimental goal is to generate rice transgenic lines which are male sterile induced by inhibition of GA biosynthesis in anther or pollen, and can be reversible to fertile plants by exogenous GA treatment. Therefore, it is essential to use anther/pollen specific promoters that can direct gene expression strictly at anther or pollen tissues during male gametophytic development in rice.

Total, twenty kinds of RNAi constructs containing six promoters combined with four RNAi trigger fragment of genes involving in rice GA biosynthesis pathway (OsGA20ox1, OsGA20ox3, OsGA3ox1, and OsGA3ox2) were constructed and introduced into rice. To compare with RNAi transformants, each promoter was also fused with GUS::GFP gene from pKGWFS7 vector and was introduced into rice to generate $T_{0}$ transgenic lines which were used as controls for the fertility evaluation.

$\mathrm{T}_{0}$ plants transformed with RNAi vectors exhibited on

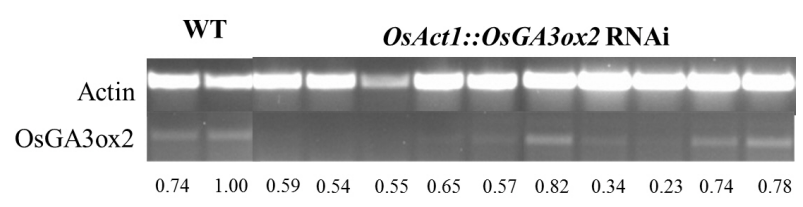

Fig. 4. RT-PCR result of OsAct1 pro-OsGA3ox2 semidwarf mutant lines. The numbers below gel photo are relative brightness value of the PCR band. 
average lower fertility than $T_{0}$ plants from control vectors carrying the anther specific promoters fused with GUS::GFP genes. Every $\mathrm{T}_{0}$ line showed lower fertility than that of wildtype rice. The average fertility rate (AFR) of $\mathrm{T}_{0}$ line was $71 \%$ on $O s c 4$ pro-GUS::GFP, $57 \%$ on $O S I P A$ proGUS::GFP, $58 \%$ on $O S S C P 1$ pro-GUS::GFP and $62 \%$ on OsAct1 pro-GUS::GFP. Furthermore, severe sterile plants, less than $10 \%$ in AFR, were not observed in the control $\mathrm{T}_{0}$ line. On the other hand, the highest AFR among RNAi lines containing genes involved in GA biosynthesis pathway was approximately $52 \%$ in $\mathrm{T}_{0}$ line of OSIPA proOsGA20ox3 in which complete-sterile plant was not found. While, the lowest AFR was $21 \%$ in OsIPA pro-OsGA3ox 2 in which 22 sterile plants were observed with the frequency of $42.5 \%$ (22/38). Among OsGA20oxl RNAi lines, a line carrying OSSCP2 promoter showed AFR of $22 \%$, but the other three lines showed $41 \%-49 \%$ AFR. It was slightly higher than other RNAi lines. In OsGA20ox3 RNAi lines, every line showed similar AFR 44\%-52\%. It was the highest AFR value among RNAi transgenic lines. Among OsGA3oxl RNAi lines, those carrying OsSCP1, OsIPA promoters, showed $26 \%$ and $28 \%$ AFR respectively, and other lines exhibited AFR range of $32 \%-45 \%$. It was the lowest AFR value among RNAi transgenic lines. In OsGA3ox 2 RNAi lines, lines controlled by OSSCP1, OsIPA promoter showed $24 \%$ and $21 \%$ AFR, and other lines showed AFR range of 37\%-51\% (Table 4).

In conclusion, AFRs in RNAi $\mathrm{T}_{0}$ lines of GA20oxl, GA20ox3, GA3ox1, and GA3ox2 were 37\%, 49\%, 32\% and $36 \%$ respectively. Severe sterile plants have been found in most populations of $T_{0}$ RNAi plants except for that of
OSIPA pro-GA20ox $3 \mathrm{~T}_{0}$ plants. Hence, our data of fertility analysis showed that all AFRs were similar, except for the GA20ox3 $\mathrm{T}_{0}$ RNAi line. Although $\mathrm{T}_{0}$ lines of GA20ox 3 exhibited the highest AFR, it was lower than any of $\mathrm{T}_{0}$ the control lines.

Among the six promoters, more effective promoters were those of OsSCP2, OSSCP1 and OsIPA. In OsGA3ox RNAi lines, $O S S C P 1$ and $O S I P A$ promoter showed more effective than other promoters. In OsGA20ox RNAi lines, unlike with OsGA3ox, OsSCP2 promoter had stronger effect than other promoters. Although fertility of RNAi $\mathrm{T}_{0}$ transgenic plants was slightly lower than transgenic control lines, numbers of glumous flowers in transgenic plants were almost similar between RNAi and control transgenic plants.

\section{Fertility of transgenic lines}

In our study, the accurate assessment of a fertility-related phenotype that occurs frequently in transgenic plants caused by somaclonal variation during the tissue-culture process was the important point. They usually showed reduced-fertility or even sterility because T-DNA insertions can generate the defects in anther development due to chromosome re-arrangements, and also because Agrobacterium-mediated transformation tends to decrease fertility frequency (Xing and Zachgo 2007). For instance, Hsing et al. (2007) have produced approximately 55,000 $\mathrm{T}_{0}$ rice transgenic lines by using Agrobacterium-mediated transformation, and $8 \%$ of them are sterile. Although our population was smaller than of previous experiment, no severe sterile phenotype was found on control transgenic plants.

Table 4. Average fertility (\%) of $\mathrm{T}_{0}$ RNAi lines from RNAi vectors of genes involving in GA biosynthesis.

\begin{tabular}{|c|c|c|c|c|c|c|}
\hline \multirow{2}{*}{ Promoters } & \multicolumn{5}{|c|}{ Genes } & \multirow{2}{*}{ Total } \\
\hline & $G U S$ & GA20ox 1 & GA20ox 3 & GA3ox 1 & GA3ox 2 & \\
\hline OsActl pro & 62 & 39 & 50 & 26 & 48 & $58^{\mathrm{a}, \mathrm{z})}$ \\
\hline Osc4 pro & 71 & - & - & 45 & 40 & $56^{\mathrm{a}}$ \\
\hline Osg6b pro & - & - & - & 32 & 52 & $58^{\mathrm{a}}$ \\
\hline OsSCP1 pro & 58 & 41 & 50 & 26 & 24 & $46^{\mathrm{b}}$ \\
\hline$O s S C P 2$ pro & - & 22 & 44 & 33 & 37 & $37^{\mathrm{c}}$ \\
\hline$O s I P A$ pro & 57 & 49 & 52 & 28 & 21 & $41^{\mathrm{bc}}$ \\
\hline Total & $61^{\mathrm{a}}$ & $37^{\mathrm{c}}$ & $49^{\mathrm{b}}$ & $32^{\mathrm{c}}$ & $36^{\mathrm{c}}$ & \\
\hline
\end{tabular}

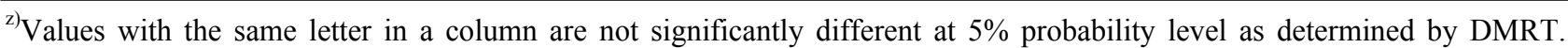




\section{Progeny analysis of RNAi lines with low fertility}

We also planted progenies of RNAi lines, including severe sterile line and normal fertility line. Through several generations $\left(T_{1}-T_{3}\right)$, RNAi transgenic lines showed segregated fertility or restore fertility. It was equally OsGA20ox and OsGA3ox lines. Undoubtedly, progenies of lower FR lines showed lower FR than higher FR lines progenies. However, in most cases transgenic lines fertility was restored. Even though their $\mathrm{T}_{0}$ line showed severe sterile phenotype (less than $5 \% \mathrm{FR}$ ) their progenies showed restored fertility up to $80 \%$; it is almost same with wildtype. In some cases, sterile phenotype was also transferred to $T_{3}$ progeny but it also showed restored fertility up to $30 \%$. Some transgenic lines lose their T-DNA through several generations and because of that their fertility was restored. Also, some lines might have their trait by somaclonal variation during rice tissue culture process; it might have disappeared through several generations.

\section{DISCUSSION}

\section{Effect of GA in rice flower development}

Many experiments have shown that the shortage of endogenous GA content causes the male-sterile phenotype. Since it has been known that GA's major function in plants is controlling stem growth and breaking dormancy, researchers have been focused on plant height especially on dwarf phenotype. Also, GA-related dwarf mutants had been used for critical genetic source of modern rice breeding. In spite of the following facts, some studies suggest that GA also controls fertility and flower development. The goal of this study is to produce a reversible male-sterility by controlling the hormones, specifically $\mathrm{GA}$ for the production of $\mathrm{F}_{1}$ hybrid rice. Traditionally in $F_{1}$ hybrid production, the three-parent system requires an MS line, maintainer line and restorer line. However, this system is not an effective way of producing $F_{1}$ hybrid seed because of its cost-ineffectiveness. By using the reversible male-sterile line, the 2-parent system, which requires an MS line and a restorer line, can be employed. The future goal of this study is to focus on examination of fertility recovery by exogenous GA treatment.

Among GA-biosynthesis genes, OsGA20ox3 and OsGA3ox 1 predominantly express gene at floral tissues and OsGA20ox 1 and OSGA3ox 2 express at both vegetative tissues and floral tissues. For this reason, we focused on OsGA20ox3 and OsGA3oxl as a candidate gene of GA biosynthesis regulative gene at floral tissues. Additionally, OsGA20ox 1 and OsGA3ox2 were reported as genes that are concerned with plant height in rice and their expression was detected on flower tissues. In our study, the most effective gene that might control the fertility of rice flower was the OsGA3oxl. The second most effective gene was OsGA3ox2. The OsGA20ox group was less effective than of $O s G A 3$ ox group. It is thought that there are four kinds of genes in the OsGA20ox group and so each gene expresses complementarily during the flower development stage, even though OsGA20ox3 is preferential gene. Actually, every $O s G A 20 o x$ gene family and $O s G A 3 o x$ gene family has expression at the flower, with different expression intensity. In OsGA3ox group, two genes exist and both have an important role in flower development stage. Among anther specific promoters used to induce malesterility in this study, OSSCPI and OSIPA promoters were the most effective when used together with OsGA3ox gene family, while $O S S C P 2$ promoter was the most effective when used together with OSGA20ox gene family. Actually, OSSCP genes constitute a gene family and was reported to show same expression pattern. RNAi lines with promoters of $O S S C P 1$ and $O s S C P 2$ showed small different results, indicating that both promoters are effective for reducing expression levels of OsGA20ox and OsGA3ox genes.

Male-sterility is an essential component for the successful production of $F_{1}$ hybrid rice seed. Many crops have traditionally been developed through a three-line system: the cytoplasmic genic male-sterile (CGMS) line, maintainer line, and restorer line. However, these systems have disadvantages, such as a limitation on restorer germplasm, a complex procedure for parental seed production, and time-consuming breeding of CGMS (Virmani 1994). China and several other Asian countries scientists have recently found out a new technological approach to replace the traditional CGMS system and simplified two-line protocol was successfully established, 
variety of germplasms are available as parents which maintainer lines did not need (Virmani 1994). The new hybrid production system is based on environmentsensitive a genetic male-sterile (EGMS) line which is controlled by environmental condition like photoperiod or temperature (Virmani 1994). One weakness point of EGMS lines is that environmental conditions must be constant. Therefore, unexpected environmental changes can cause of fertility restoration that lead to failure in hybrid seed production.

Recoverable male sterility by chemical treatment can be a useful and crucial tool for hybrid seed production. Most male-sterile mutants produce defective in pollen generation or showed abnormal developmental process in sporophytic tissue in the tapetum and filaments. Other developmental processes are related to changes in the levels of plant growth regulator (Huang et al. 2003). For instance, the need for gibberellins in formation of male gametophytes was supported by studies of defective mutants that can restore the male sterility through application of GAs (Jacobsen and Olszewski 1991). Although endogenous phytohormones modulation can cause male sterility in many higher plants, their fertility can be recovered with exogenous application of suitable hormones.

Here we report that sterility or reduced fertility in rice can be induced through the controlling of endogenous GA biosynthesis by using OsGA20ox and OsGA3ox RNAi vector. Therefore, these results suggest the useful biotechnological approaches to engineer male sterility for the widespread use of hybrid production in self-pollinated crops.

\section{ACKNOWLEDGEMENTS}

This work was carried out with the support of the "Cooperative Research Program for Agriculture Science \& Technology Development (Agricultural Biotechnology Research Center. Project No. 01369001)" Rural Development Administration, Republic of Korea.

\section{REFERENCES}

Chhun T, Aya K, Asano K, Yamamoto E, Morinaka Y, Watanabe M, et al. 2007. Gibberellin regulates pollen viability and pollen tube growth in rice. Plant Cell 19: 3876-3888.

Deveshwar P, Bovill WD, Sharma R, Able JA, Kapoor S. 2011. Analysis of anther transcriptomes to identify genes contributing to meiosis and male gametophyte development in rice. BMC Plant Biol. 11: 78.

Endo M, Tsufchya T, Saito H, Matsubara H, Hakozaki H, Masuko $\mathrm{H}$, et al. 2004. Identification and molecular characterization of novel anther-specific genes in Oryza sativa L. by using cDNA microarray. Genes Genet. Syst. 79: 213-226.

Fujita M, Horiuchi Y, Ueda Y, Mizuta Y, Kuibo T, Yano K, et al. 2010. Rice expression atlas in reproductive development. Plant Cell Physiol. 51: 2060-2081.

Gubler F, Kalla R, Roberts JK, Jacobsen JV. 1995. Gibberellin-regulated expression of a $m y b$ gene in barley aleoruone cells: evidence for Myb transactivation of a high-pl $\alpha$-amylase gene promoter. Plant Cell 7: 1879-1891.

Gupta V, Khurana R, Tyagi AK. 2007. Promoters of two anther-specific genes confer organ-specific gene expression in a stage-specific manner in transgenic systems. Plant Cell Rep. 26: 1919-1931.

Hirano K, Aya K, Hobo T, Sakakibara H, Kojima M, Shim RA, et al. 2008. Comprehensive transcriptome analysis of phytohormone biosynthesis and signaling genes in microspore/pollen and tapetum of rice. Plant Cell Physiol. 49: 1429-1450.

Hsing YI, Chern CG, Fan MJ, Lu PC, Chen KT, Lo SF, et al. 2007. A rice gene activation/knock mutant resource for high throughput functional genomics. Plant Mol. Biol. 63: $351-364$

Huang S, Cerny RE, Qi Y, Bhat D, Aydt CM, Hason DD, et al. 2003. Transgenic studies on the involvement of cytokinin and gibberellin in male development. Plant Physiol. 131: 1270-1282.

Itoh H, Ueguchi-Tanaka M, Sentoku N, Kitano H, Matsuoka M, Kobayashi M. 2001. Cloning and functional analysis of gibberellin 3 $\beta$-hydroxylase genes that differently expressed during the growth of rice. Proc. Natl. Acad. Sci. U.S.A. 98: 8909-8914.

Itoh H, Ueguchi-Tanaka M, Sakamoto T, Kayano T, Tanaka 
H, Ashikari M, et al. 2002. Modification of rice plant height by suppressing the height-controlling gene, D18, in rice. Breed. Sci. 52: 215-218.

Itoh J, Nonomura K, Ikeda K, Yamaki S, Inukai Y, Yamagishi $\mathrm{H}$, et al. 2005. Rice plant development: from zygote to spikelet. Plant Cell Physiol. 46: 23-47.

Jacobsen SE, Olszewski NE. 1991. Characterization of the arrest in anther development associated with gibberellins deficiency of the gib-1 mutant of tomato. Plant Physiol. 97: 409-414.

Kaneko M, Inukai Y, Ieguchi-Tanaka M, Itoh H, Izawa T, Kobayashi Y, et al. 2003. Loss-of-function mutations of the rice $G A M Y B$ gene impair $\alpha$-amylase expression in aleurone and flower development. Plant Cell 16: 33-44.

Koornneef M, van der Veen JH. 1980. Induction and analysis of gibberellin sensitive mutants in Arabidopsis thaliana (L.) heynh. Theor. Appl. Genet. 58: 257-263.

Ma H. 2005. Molecular genetic analyses of microsporogenesis and microgametogenesis in flowering plants. Annu. Rev. Plant Biol. 56: 393-434.

McElroy D, Zhang W, Cao J, Wu R. 1990. Isolation of an efficient actin promoter for use in rice transformation. Plant Cell 2: 163-171.

Oikawa T, Koshioka M, Kojima K, Yoshida H, Kawata M. 2004. A role of OsGA20ox1, encoding an isoform of gibberellin-20-oxidase, for regulation of plant stature in rice. Plant Mol. Biol. 55: 687-700.

Park JL, Hakozaki H, Endo M, Takada Y, Ito H, Uchida M, et al. 2006. Molecular characterization of mature pollenspecific genes encoding novel small cysteine-rich proteins in rice (Oryza sativa L.). Plant Cell Rep. 25: 466-474.

Qiao F, Yang Q, Wang CH, Fan YL, Wu XF, Zhao KJ. 2007. Modification of plant height via RNAi suppression of OsGA20ox2 gene in rice. Euphytica 158: 35-45.

Ronald PC, Chen DH. 1999. A rapid DNA minipreparation method suitable for AFLP and other PCR applications. Plant Mol. Biol. Rep. 17: 53-57.

Sakamoto T, Kobayashi M, Itoh H, Tagiri A, Kayano T, Tanaka H, et al. 2001. Expression of a gibberellin 2-oxidase gene around the shoot apex is related to phase transition in rice. Plant Physiol. 125: 1508-1516.

Sakamoto T, Miura K, Itoh H, Tatsumi T, Ueguchi-Tanaka
M, Ishiyama K, et al. 2004. An overview of gibberellin metabolism enzyme genes and their related mutants in rice. Plant Physiol. 134: 1642-1653.

Spielmeyer W, Ellis MH, Chandler PM. 2002. Semidwarf $(s d-1)$, "green revolution" rice, contains a defective gibberellin 20-oxidase gene. Proc. Natl. Acad. Sci. U.S.A. 99: 9043-9048.

Suwabe K, Suzuki G, Takahashi H, Shiono K, Endo M, Yano $\mathrm{K}$, et al. 2008. Separated transcriptomes of male gametophyte and tapetum in rice: validity of a laser microdissection (LM) microarray. Plant Cell Physiol. 49: 1407-1416.

Tang X, Zhang ZY, Zhang WJ, Zhao XM, Li X, Zhang D, et al. 2010. Global gene profiling of laser-captured pollen mother cells indicates molecular pathways and gene subfamilies involved in rice meiosis. Plant Physiol. 154: 1855-1870.

Toki S, Hara N, Ono K. 2006. Early infection of scutellum tissue with Agrobacterium allows high-speed transformation of rice. Plant J. 47: 969-976.

Yoshida S, Forno DA, Cock JH, Gomez KA. 1976. Laboratory manual for physiological studies of rice. International Rice Research Institute. Manila, Philippines. pp. 61-66.

Tsuchiya T, Toriyama K, Nasrallah ME, Ejiri S. 1992. Isolation of genes abundantly expressed in rice anthers at the microspore stage. Plant Mol. Biol. 20: 1189-1193.

Tsuchiya T, Toriyama K, Ejiri S, Hinata K. 1994. Molecular characterization of rice genes specifically expressed in the anther tapetum. Plant Mol. Biol. 26: 1737-1746.

Virmani SS. 1994. Heterosis and hybrid rice breeding. Monograph on theoretical and applied genetics. Vol. 22. Springer-Verlag, Berlin Heidelberg.

Yamaguchi T, Lee DY, Miyao A, Hirochika H, An G, Hirano H. 2006. Functional diversification of the two C-Class MADS Box genes OSMADS3 and OSMADS58 in Oryza sativa. Plant Cell 18: 15-28.

Xing S, Zachgo S. 2007 Pollen lethality: a phenomenon in Arabidopsis RNA interference plants. Plant Physiol. 145: 330-333.

Zhang DB, Wilson ZA. 2009. Stamen specification and anther development in rice. Chinese Sci. Bull. 54: 2342-2353. 